\title{
On-Line Accuracy Assessment for the Dual Six-Port ANA: Statistical Methods for Random Errors
}

\author{
ROBERT M. JUDISH AND GLENN F. ENGEN
}

\begin{abstract}
A basic property of a measurement process is that repeated observations of the same quantity will not give identical results due to the presence of random errors. In order to assess the effects of random errors in our measurement process, we need to build in redundancy. This paper presents a brief summary of the statistical methods used to evaluate the random errors in dual six-port measurements of reflection coefficient and scattering parameters.
\end{abstract}

\section{INTRODUCTION}

A companion paper has introduced the six-port and the model equations describing its operation [1]. There it was mentioned that the six-port accuracy is a function of the parameter being measured, the 11 parameters that characterize the six-port, and the errors in their determination. In this paper we will discuss the procedures for assessing the effect of random errors in six-port measurement of reflection coefficient and scattering parameters.

It is well known that repeated observations of the same quantity will not give identical results due to the presence of errors. If these errors are random in nature, and can be described by a probability distribution, then we can use statistical procedures to estimate the most likely or probable value for the true value of the quantity of interest, given our collection of observations. This process of determining parameter values, with statistical considerations of the errors in mind, is called statistical parameter estimation.

In the six-port environment, we have a collection of power meter observations and (in the calibration mode) the first objective is to estimate the parameters that characterize the $P-w$ transform. In this phase we also treat the $w$ 's which result from the different excitation conditions as unknown parameters to be estimated. Following this, we treat these estimated w's as a collection of observations from which we will estimate the parameters of the $w-\Gamma$ transformation. In addition, we obtain estimates of the errors in the parameter estimates.

These statistical procedures are discussed in detail as they are applied in the three phases involved in calibrating the dual six-ports. Subsequent application of propagation of error formulas give us the uncertainty in the main parameters of interest, namely, reflection coefficient and attenuation.

Manuscript received June 23, 1987.

The authors are with the National Bureau of Standards, Electromagnetic Fields Division, Boulder, CO 80303.

IEEE Log Number 8714045.

\section{Statistical Parameter Estimation}

Before discussing the application of the statistical methods to the problem at hand, we will look at two elementary examples that contain most of the concepts which will be used.

To be explicit, we shall first consider the simple case of repeated observations of resistance using an ohmmeter. Let $\rho$ denote the "true" resistance, and $R_{i}$ the observed value for the $i$ th measurement. Each $R_{i}$ is equal to the "true" value plus a random error which we denote by $e_{i}$. This can be expressed by the following statistical model:

$$
R_{i}=\rho+e_{i} \quad i=1, \cdots, n .
$$

We further assurne that the $e_{i}$ are statistically independent and can be described by a probability distribution with a mean value of $\delta$ and variance $\sigma^{2}$. The parameters $\delta$ and $\sigma^{2}$ are properties of the ohmmeter. For an ideal ohmmeter these parameters would both be zero and a single observation $R_{i}$, would determine $\rho$ without error. In real life, both $\delta$ and $\sigma^{2}$ are nonzero which limits the accuracy to which $\rho$ can be determined.

If $\delta=0$, and $\sigma^{2} \neq 0$, the measuring instrument is "unbiased." Thus the $R_{i}$ will tend to cluster around the true value. If $\delta \neq 0$ we have a "systematic error" whose presence cannot be detected by repeated measurements, and whose evaluation lies outside the scope of the present discussion. In what follows, we shall assume $\delta=0$. It remains to consider the effect of $\sigma^{2}$ on the accuracy with which $\rho$ can be determined. In practice, two questions are, given a collection of $n$ observations, $\left.R_{1}, \cdots, R_{n} ; 1\right)$ how do we obtain or estimate the most probable value of $\rho$; and 2) how do we characterize the error in our estimate?

Provided that the distribution function for the $e_{i}$ is Gaussian, the most probable value of $\rho$ is given by the least squares estimation procedure which is also widely used for non-Gaussian distributions. In what follows we do not make the assumption that the distribution function is Gaussian. The least squares estimation procedure chooses the value for the parameter $\rho$ that minimizes the sum of the squared errors. The error is given by $e_{i}=R_{i}$ $-\rho$ and the sum of squared errors is

$$
\phi(\rho)=\sum e_{i}^{2}=\sum\left(R_{i}-\rho_{i}\right)^{2} .
$$

Here, $\phi(\rho)$ is a function of $\rho$ and the value of $\rho$ which minimizes (2) is the least squares estimator and is denoted with a "^" over it, i.e., $\hat{\rho}$. The solution is obtained by 
equating the derivative of $\phi$ to zero and solving for $\rho$. The residuals are defined as the difference between each observation and the estimated value. These residuals provide estimates of the errors in each of the observed $R_{i}$. The estimated variance of the errors is obtained by dividing the sum of squared residuals by $n-1$ and is denoted by $\hat{\sigma}^{2}$ where

$$
\hat{\sigma}^{2}=\frac{\sum\left(R_{i}-\hat{\rho}\right)^{2}}{n-1} .
$$

The estimated standard deviation is given as the square root of the quantity in (3). We have seen that the estimate for $\rho$ is obtained as the minimization to the quadratic function in (2). The estimate $\hat{\rho}$ can be shown to be the arithmetic average of the observed $R_{i}$. It can further be shown [2] that the variance of $\hat{\rho}$, denoted by $V(\hat{\rho})$, is

$$
V(\hat{\rho})=\frac{\sigma^{2}}{n} .
$$

The estimated variance of $\hat{\rho}$ is obtained by using the estimated value for $\sigma^{2}$ from (3) in (4). The positive square root gives the estimated standard deviation of $\hat{\rho}$. The standard deviation of $\hat{\rho}$ is often called the standard error. We now have an estimate of the error in $\hat{\rho}$ which results from our estimation procedure and errors in the observations.

In the previous example, the observed $R_{i}$ is ideally the parameter $\rho$ whose value we seek. To further illustrate the statistical methods, it is useful to consider a second example which is a simplified version of one of the actual problems to be solved. A complex parameter $w$ is to be determined from the intersection of three circles in the complex plane where the circle centers are given and the observed quantities $R_{i}^{2}$ are the squares of the radii. Here one has

$$
R_{i}^{2}=\left|w-c_{i}\right|^{2} \quad i=1, \cdots, 3
$$

where the circle centers are denoted by $c_{i}$.

Although there may be only a single observation for each of the $R_{i}^{2}$, it will not be possible, in general, to find a value of $w$ which satisfies all three equations because of measurement error in $R_{i}^{2}$. As before we can define an error $e_{i}$, such that

$$
e_{i}=R_{i}^{2}-\left|w-c_{i}\right|^{2}
$$

and define a function

$$
\phi(w)=\sum_{i=1}^{3} e_{i}^{2}
$$

which may be minimized with respect to $w$, leading to the estimate $\hat{w}$ for the "true" value of $w$.

The foregoing procedure is appropriate if the standard deviations of the $e_{i}$, which are now characteristics of different measuring instruments, are equal. In the more general case, where this is not true, (7) is replaced by

$$
\phi(w)=\sum_{i=1}^{3} \frac{e_{i}^{2}}{\sigma_{i}^{2}}
$$

where $\sigma_{i}$ is the standard deviation of the $e_{i}$. The function in (8) is a "weighted" sum of squares that is minimized for $w$. A further generalization of this basic idea will be developed in the context of the specific application. It remains to assess the variance of $\hat{w}$. This problem is considered in Appendix B.

The statistical estimation procedures that we have outlined above are applied in assessing the effects of random errors in the six-port. As we shall see the six-port equations are nonlinear and the errors do not belong to a single probability distribution. Thus we will need to modify the estimation procedures. We shall adapt the statistical methods in the following sections.

\section{Estimation of the Six-Port Parameters}

\section{A. P-w Transformation}

The model equations describing the $P-w$ transformation [1] are given by

$$
\begin{aligned}
R_{i j} & =\rho_{i}\left|w_{j}-\lambda_{i}\right|^{2}+e_{i j}, \\
i & =1, \cdots, 3, \quad j=1, \cdots, 16
\end{aligned}
$$

where $R_{i j}$ is the ratio of the observed power readings $P_{i j}$ to $P_{0 j}^{i} ; \rho_{i}$ and $\lambda_{i}$ are parameters of the six-port with $\rho_{1}=$ 1 and $\lambda_{1}=\operatorname{Im}\left(\lambda_{2}\right)=0 ; w_{j}$ are the complex unknowns as described in [1]; and $e_{i j}$ is the random error in the observed ratio $R_{i j}$. We use the notation $P_{0 j}^{i}$ to denote the "reference" sidearm power that is read with the $i$ th sidearm $P_{i j}$. Since $R_{i j}$ contains a different $P_{0}$ for each sidearm reading we assume each $e_{i j}$ is uncorrelated with each other. We also assume the $e_{i j}$ has a mean of zero and a variance $\sigma^{2} r_{i j}^{2}$ where $r_{i j}$ is the "true" power ratio. Thus the variance of $e_{i j}$ is not constant but is proportional to the power ratio squared. It is convenient to write (9) in matrix form as

$$
\boldsymbol{R}_{j}=\boldsymbol{f}_{j}\left(w_{j}, \boldsymbol{\theta}\right)+\boldsymbol{e}_{j}, \quad j=1, \cdots, 16
$$

where

$$
\begin{aligned}
\boldsymbol{R}_{j} & =\left[\begin{array}{l}
R_{1 j} \\
R_{2 j} \\
R_{3 j}
\end{array}\right] \\
\boldsymbol{f}_{j}\left(w_{j}, \boldsymbol{\theta}\right) & =\left[\begin{array}{l}
\left|w_{j}\right|^{2} \\
\rho_{2}\left|w_{j}-\lambda_{2}\right|^{2} \\
\rho_{3}\left|w_{j}-\lambda_{3}\right|^{2}
\end{array}\right] \\
\boldsymbol{e}_{j} & =\left[\begin{array}{l}
e_{1 j} \\
e_{2 j} \\
e_{3 j}
\end{array}\right] \\
\boldsymbol{\theta} & =\left[\begin{array}{l}
\rho_{2} \\
\lambda_{2} \\
\rho_{3} \\
\operatorname{Re}\left(\lambda_{3}\right) \\
\operatorname{Im}\left(\lambda_{3}\right)
\end{array}\right] .
\end{aligned}
$$


The vector of errors $\boldsymbol{e}_{j}$ has a mean zero and covariance matrix $\sigma^{2} V_{j}$ where

$$
\boldsymbol{V}_{j}=\left[\begin{array}{ccc}
r_{1 j}^{2} & 0 & 0 \\
0 & r_{2 j}^{2} & 0 \\
0 & 0 & r_{3 j}^{2}
\end{array}\right] .
$$

The nondiagonal elements of $\boldsymbol{V}_{j}$ are zero since we assumed the errors were uncorrelated while the diagonal elements when multiplied by $\sigma^{2}$ give the variance of $e_{i j}$. The sum of squares that is minimized is weighted by $V_{j}^{-1}$. In practice, we do not know the true power ratios so we use the observed power ratios $R_{i j}$, to which we add .01 , as the elements in $V_{j}$. The addition of this constant recognizes the finite dynamic range of the power meters. It also enhances the convergence potential for the minimization procedure when $R_{i j} \Rightarrow 0$.

At this point (9) represents a system of 48 equations, while the complex $w_{1}, \cdots, w_{16}$ represents 32 unknowns, and the elements of $\boldsymbol{\theta}$ an additional five. The least squares estimates are obtained by minimizing the "weighted" sum of squares given by

$$
\phi(\theta, w)=\sum_{j=1}^{16} \boldsymbol{e}_{j}^{\prime} \boldsymbol{V}_{j}^{-1} \boldsymbol{e}_{j}
$$

where

$$
\boldsymbol{e}_{j}=\boldsymbol{R}_{j}-f_{j}\left(w_{j}, \boldsymbol{\theta}\right)
$$

and

$$
\boldsymbol{w}^{\prime}=\left[w_{1}, w_{2}, \cdots, w_{16}\right] .
$$

Given the structure of $V_{j}$ in (11), (12) can be written

$$
\phi(\boldsymbol{\theta}, \boldsymbol{w})=\sum_{j=1}^{16} \sum_{i=1}^{3} \frac{e_{i j}^{2}}{\left(R_{i j}+.01\right)^{2}} .
$$

The solution for the parameters in (9) is found by differentiating $\phi(\theta, w)$ with respect to $\theta$ and $\boldsymbol{w}$, setting the resulting equations to zero and solving for the unknown parameters. However, these equations are nonlinear in the parameters and cannot be solved analytically, thus requiring a numerical solution. The values that minimize (12) are the least squares estimates and are denoted by $\hat{\theta}$ and $\hat{w}$. The details of the numerical solution are found in Appendix A.

Furthermore, an estimate of the standard deviation of the errors in the power-meter ratio is obtained by evaluating (12) using $\hat{\boldsymbol{\theta}}$ and $\hat{\boldsymbol{w}}$ and dividing by the degrees of freedom which is the number of observations (48) minus the number of estimated parameters (37). This standard deviation is estimated by $\hat{\sigma}$ where

$$
\hat{\sigma}=\sqrt{\frac{\phi(\hat{\boldsymbol{\theta}}, \hat{\boldsymbol{w}})}{11}} .
$$

No parameter estimation scheme is complete unless it furnishes information about the error of estimation. Such information is provided by the covariance matrix of the parameter estimates. This covariance matrix is a square matrix with dimensions equal to the number of parameters. The variance of the $i$ th parameter is in the $i, i$ location and the covariance between the $i$ th and $j$ th parameters is in the $i, j$ location. The covariance matrix of the estimated parameters is the multivariate analog of the variance of a univariate parameter as discussed in Section I.

The estimated covariance matrix of the 37 parameters obained from the model in (9) is

$$
\operatorname{cov}\left[\begin{array}{l}
\hat{\boldsymbol{\theta}} \\
\hat{\boldsymbol{w}}
\end{array}\right]=\hat{\sigma}^{2}\left(\boldsymbol{J}^{\prime} \boldsymbol{V}^{-1} \boldsymbol{J}\right)^{-1}
$$

where $V$ is the $48 \times 48$ block diagonal matrix with submatrices $V_{j}$ and $J$ is the $48 \times 37$ Jacobian matrix containing the partial derivatives of $f_{j}$ with respect to $\theta$ and $\boldsymbol{w}$. The derivation of (15) is given in Appendix B. In what follows it is convenient to partition this covariance matrix as

$$
\operatorname{cov}\left[\begin{array}{l}
\hat{\boldsymbol{\theta}} \\
\hat{\boldsymbol{w}}
\end{array}\right]=\left[\begin{array}{ll}
\boldsymbol{\Sigma}_{\theta} & \boldsymbol{C} \\
\boldsymbol{C}^{\prime} & \boldsymbol{\Sigma}_{\boldsymbol{w}}
\end{array}\right]
$$

where $\boldsymbol{\Sigma}_{\theta}$ is the $5 \times 5$ covariance matrix of $\hat{\boldsymbol{\theta}}$, and $\boldsymbol{\Sigma}_{w}$ is the $32 \times 32$ covariance matrix of $\hat{\boldsymbol{w}}$, and $\boldsymbol{C}$ is the $5 \times$ 32 matrix containing the covariances between the elements in $\hat{\boldsymbol{\theta}}$ and $\hat{\boldsymbol{w}}$.

The estimation procedure described above is applied to each network of the dual six-port independently and we use subscripts to denote the results for each six-port. We therefore obtain parameter estimates $\hat{\boldsymbol{\theta}}_{1}$ and $\hat{\boldsymbol{\theta}}_{2}$ and their covariance matrices $\boldsymbol{\Sigma}_{\theta_{1}}$ and $\boldsymbol{\Sigma}_{\theta_{2}}$. We also obtain estimates of the $w$ values $\hat{\boldsymbol{w}}_{1}$ and $\hat{\boldsymbol{w}}_{2}$ and their covariance matrices $\Sigma_{w_{1}}$ and $\boldsymbol{\Sigma}_{w_{2}}$.

\section{B. Scattering Parameters of the Thru, Reflect, and Line Two-Ports}

As outlined in the companion paper [1] and using Engen's notation, the next task is the estimate the $T_{i j}, R_{i j}$, and $L_{i j}$ which characterize the thru, reflect, and line twoports, respectively. The estimated $w_{1}$ and $w_{2}$ values (for the thru excitation) obtained in the prior section now become the "observations" which are functionally related to the scattering parameters for the thru two-port by the following equations:

$$
\begin{aligned}
& w_{1 i}=T_{11}+T_{12} K_{i} \\
& w_{2 i}=T_{22}+T_{12} / K_{i}, \quad i=1, \cdots, 4
\end{aligned}
$$

where the $K_{i}$ are "nuisance" parameters whose values are determined by the excitation conditions and which are estimated along with the $T_{i j}$ as part of the solution, although they are of no further interest.

In matrix form (17) may be written as

$$
w=f(T, K)+g
$$

where $w$ is a $16 \times 1$ vector which contains the real and imaginary components of the "observed" $w_{i j}, f$ is a vector of functions containing the right-hand side of (17), $\boldsymbol{T}$ 
and $\boldsymbol{K}$ are vectors with elements $T_{i j}$ and $K_{i}$, respectively, and $g$ is a vector of errors in the observed $w$ values. As before, one forms an objective function

$$
\phi(T, K)=g^{\prime} V_{g}^{-1} g
$$

which is then minimized with respect to $\boldsymbol{T}$ and $\boldsymbol{K}$ to obtain the estimates $\hat{T}$. (As noted before, estimates for $K$ are also obtained but discarded.) The associated covariance matrix of $T, \mathbf{\Sigma}_{T}$, is also obtained as explained in Appendix B. The matrix $V_{g}$, whose inverse appears in (19), is

$$
V_{g}=\left[\begin{array}{cc}
\boldsymbol{\Sigma}_{w_{1_{1}}} & \mathbf{0} \\
\mathbf{0} & \boldsymbol{\Sigma}_{w_{2}}
\end{array}\right]
$$

where $\boldsymbol{\Sigma}_{w_{1}}$ and $\boldsymbol{\Sigma}_{w_{2}}$ are the submatrices of $\boldsymbol{\Sigma}_{w_{1}}$ and $\boldsymbol{\Sigma}_{w_{2}}$ in (16), respectively, corresponding to the $w$ values for the thru excitation.

The estimates for $R_{i j}$ and $L_{i j}$ are obtained in a similar manner, although as explained in [1], $1 / w_{1}$ is taken as the observed quantity for the reflect instead of $w_{1}$ and this calls for a transformation of the associated covariance matrix to obtain $V_{g}$. If the only source of error was from the power meters, then one would expect $\phi_{\min } / 2$ to be unity. Values much greater than one indicate other error sources.

As a rule the results obtained for the thru and line twoports agree with expectation, while those for the reflect two-port indicate the presence of another error source, which may be identified as a failure to satisfy the $\Gamma_{1}=$ $\Gamma_{2}$ postulate due to nonideal connector behavior. Indeed, the error from this source may be much larger than that due to $w$.

In order to deal with this problem we have reformulated the model where we assume the $w$ values to be free of error and the observed inconsistencies are due to a failure to satisfy the $\Gamma_{1}=\Gamma_{2}$ condition. Instead of (18), an alternative formulation leads to

$$
\boldsymbol{\Gamma}_{1}-\boldsymbol{\Gamma}_{2}=\boldsymbol{h}(\boldsymbol{w}, \boldsymbol{R})
$$

where $\boldsymbol{h}$ is a vector of errors. The objective function now becomes

$$
\phi(\boldsymbol{R})=\boldsymbol{h}^{\prime} \boldsymbol{V}_{h}^{-1} \boldsymbol{h}
$$

and $\boldsymbol{V}_{h}$ is proportional to the covariance matrix of $\boldsymbol{h}$. $\boldsymbol{V}_{\boldsymbol{h}}$ is inferred from engineering considerations of the nonideal connector performance. The solution for this formulation provides an alternative estimate of $\boldsymbol{R}$ and its covariance matrix which is used in lieu of that previously obtained.

Provided that the connection is stable, the determination of the scattering parameters $\boldsymbol{T}$ and $\boldsymbol{L}$, for the thru and line two-ports will be insensitive to conncitor errors. In order to assess the effects of connector errors it is necessary to repeat the estimation of $\boldsymbol{T}$ and $\boldsymbol{L}$ subsequent to breaking and remaking the connection. After this has been done, one can average the results to obtain the "best" estimate for $\boldsymbol{T}$ and $\boldsymbol{L}$. Provided that the number of repeat observations is seven or more, one could estimate the associated covariance matrix from the sample, but this amount of data is not ordinarily available. As an alternative, one can use the variation in the results to estimate certain parameters associated with an assumed model for the nonideal connector performance which has been developed from engineering considerations. This has proven moderately successful in explaining the observed results and promises to become more so with further experimental and theoretical study.

\section{C. $w-\Gamma$ Transformation}

In the previous section we obtained the scattering parameters associated with the reflect, thru, and line twoports. The estimates, $R_{11}, R_{12}, \cdots, L_{22}$ now become a collection of observations which are funtionally related to the Error-Box parameters [1], $\alpha_{1}, \beta_{1}, \gamma_{1}, \cdots, \gamma_{2}$ and $e^{-2 \gamma^{\prime}}$. Equations (17)-(25) in [1] give the functional relationship which involves nine complex observations and seven complex parameters. In matrix form these equations can be written as

$$
\boldsymbol{S}=\boldsymbol{f}\left(\xi_{1}, \xi_{2}, \gamma l\right)+\boldsymbol{d}
$$

where $S$ is an $18 \times 1$ vector of the scattering parameters, $R_{11}, \cdots, L_{22}, f(\cdot)$ contains the right-hand side of (17)(25) in [1], $\xi_{1}$ and $\xi_{2}$ are the Error-Box parameters for six-ports 1 and 2 , respectively, and $\gamma$ is the propagation constant for the line, and $l$ its length. The vector $d$ represents the errors in $\boldsymbol{S}$ and we assume the covariance of $\boldsymbol{d}$ is proportional to $V_{d}$ where

$$
\boldsymbol{V}_{d}=\left[\begin{array}{ccc}
\boldsymbol{\Sigma}_{R R} & \mathbf{0} & \mathbf{0} \\
\mathbf{0} & \boldsymbol{\Sigma}_{T T} & \mathbf{0} \\
\mathbf{0} & \mathbf{0} & \boldsymbol{\Sigma}_{L L}
\end{array}\right] .
$$

The submatrices $\Sigma_{R R}, \Sigma_{T T}, \Sigma_{L L}$, in $\boldsymbol{V}_{d}$ are the covariance matrices we obtain for the estimated scattering parameters for the reflect, thru, and line two-ports, respectively.

The sum of squares we minimize is

$$
\phi\left(\xi_{1}, \xi_{2}, \gamma l\right)=\boldsymbol{d}^{\prime} \boldsymbol{V}_{d}^{-1} \boldsymbol{d} .
$$

The least squares estimates and covariance matrix of the estimates are obtained using the methods in Appendixes $\mathrm{A}$ and $\mathrm{B}$. The parameter estimates are denoted by $\hat{\xi}_{1}, \hat{\xi}_{2}$, and $\hat{\gamma} l$, while the corresponding covariance matrix we obtain is partitioned as

$$
\operatorname{cov}\left[\begin{array}{l}
\hat{\xi}_{1} \\
\hat{\xi}_{2} \\
\hat{\xi}_{3}
\end{array}\right]=\left[\begin{array}{lll}
\boldsymbol{\Sigma}_{\xi_{1}} & \boldsymbol{\Sigma}_{12} & \boldsymbol{\Sigma}_{1_{\gamma /}} \\
\boldsymbol{\Sigma}_{12} & \boldsymbol{\Sigma}_{\xi_{2}} & \boldsymbol{\Sigma}_{2 \gamma /} \\
\boldsymbol{\Sigma}_{1_{\gamma l}} & \boldsymbol{\Sigma}_{2 \gamma^{\prime}} & \boldsymbol{\Sigma}_{\gamma l}
\end{array}\right] .
$$

The covariances of the parameter vectors $\hat{\xi}_{1}, \hat{\xi}_{2}$, and $\hat{\gamma} l$ are the diagonal submatrices in (26), while the off-diagonal submatrices contain the covariances between the different parameter vectors. For example $\boldsymbol{\Sigma}_{\xi_{1}}$ and $\boldsymbol{\Sigma}_{\xi_{2}}$ are the covariance matrices of $\hat{\xi}_{1}$ and $\hat{\xi}_{2}$, respectively, and $\Sigma_{12}$ contains the covariances between the estimates in $\hat{\xi}_{1}$ and $\hat{\xi}_{2}$, etc. 


\section{Measurement of $\Gamma$}

The prior discussion focused on the methods for estimating the parameters that characterize the dual six-port. This process of estimation is referred to as the six-port calibration. In this section we discuss the procedure for estimating the reflection coefficient $\Gamma$ for an unknown oneport device.

\section{A. P-w Transformation}

When an unknown is connected to the test port, the response of the six-port is the power meter observations; $P_{0 u}^{i}, P_{i u}, i=1, \cdots, 3$ where the subscript $u$ denotes the excitation condition for the unknown. The model relating the ratios of these power observations is given by

$$
R_{i u}=\hat{\rho}_{i}\left|w_{u}-\hat{\lambda}_{i}\right|^{2}+e_{i u}, \quad i=1,2,3 .
$$

This model is equivalent to (9) except that it is written for a single excitation condition (subscript $j=u$ ). Furthermore, we assume the six-port parameters to be the estimates $\hat{\rho}_{i}$ and $\hat{\lambda}_{i}$. Equation (27) involves three observations $\left(R_{1 u}, R_{2 u}, R_{3 u}\right)$ and two unknown parameters (complex $\left.w_{u}\right)$.

The solution for the unknown parameter $w_{u}$ is obtained in the same manner as before. The sum of squares to be minimized is

$$
\phi\left(w_{u}\right)=\sum_{i=1}^{3} \frac{e_{i u}^{2}}{\left(R_{i u}+.01\right)^{2}}=\sum_{i=1}^{3}\left(\frac{R_{i u}-\hat{\rho}\left|w_{u}-\hat{\lambda}_{i}\right|^{2}}{\left(R_{i u}+.01\right)^{2}}\right) .
$$

Notice that we write the sum of squares in (28) as a function of $w_{u}$ only. This means that we treat $\hat{\boldsymbol{\theta}}$ as fixed and $w_{u}$ as the only unknown parameter.

We also obtain another estimate of the standard deviation of the errors in the power ratios (see (14)). Although this later estimate is based on only a single degree of freedom, it does provide a monitor of the performance of the power meters. It is beyond the scope of this paper to discuss the statistical control procedures used as these will be published in a later article.

Although the solution we obtain here closely parallels an earlier treatment [3], it is important to realize that in addition to getting an estimate $\hat{w}_{u}$, the current formulation allows us to assess the errors in $\hat{w}_{u}$ that result from the power-meter errors and errors in the parameter estimates $\hat{\rho}_{i}$ and $\hat{\lambda}_{i}$.

\section{B. Uncertainty of $\hat{w}_{u}$}

The solution for $w_{u}$ is obtained by minimizing (28). There we assumed the values for $\hat{\boldsymbol{\theta}}$. However if we use other values for the parameter vector, say $\tilde{\boldsymbol{\theta}}$, we will get another solution, say $\tilde{w}_{u}$. Thus $\hat{\mathrm{w}}_{u}$ and $\tilde{w}_{u}$ are two estimates of the true $w_{u}$ that differ due to differences between $\hat{\boldsymbol{\theta}}$ and $\tilde{\boldsymbol{\theta}}$. The covariance matrix of $\hat{w}_{u}$ therefore needs to reflect the uncertainty in $\hat{\boldsymbol{\theta}}$.

The function in (28) that we minimize depends on the observations, $\boldsymbol{R}_{i u}$ and the parameter estimates $\hat{\boldsymbol{\theta}}$. We therefore write $(28)$ as $\phi\left(w_{u}, \boldsymbol{R}_{u}, \hat{\boldsymbol{\theta}}\right)$ to indicate this de- pendence. At minimum we must have

$$
\frac{\partial \phi\left(\hat{w}_{u}, \boldsymbol{R}_{u}, \hat{\boldsymbol{\theta}}\right)}{\partial w_{u}}=0 .
$$

If we used $\boldsymbol{R}_{u}+\delta \boldsymbol{R}$ and $\hat{\boldsymbol{\theta}}+\delta \boldsymbol{\theta}$ in place of $\boldsymbol{R}_{u}$ and $\hat{\boldsymbol{\theta}}$, respectively, then this would cause our minimum to shift from $\hat{w}_{u}$ to $\hat{w}_{u}+\delta w$ where we must also have

$$
\frac{\partial \phi\left(\hat{w}_{u}+\delta w, \boldsymbol{R}_{u}+\delta \boldsymbol{R}, \hat{\boldsymbol{\theta}}+\delta \boldsymbol{\theta}\right)}{\partial w_{u}}=0 .
$$

Expanding (30) in Taylor series retaining only first-order terms gives

$$
\begin{array}{r}
\frac{\partial \phi\left(\hat{w}_{u}+\delta w, \boldsymbol{R}_{u}+\delta \boldsymbol{R}, \hat{\boldsymbol{\theta}}+\delta \boldsymbol{\theta}\right)}{\partial w_{u}} \\
\approx \frac{\partial \phi\left(\hat{w}_{u}, \boldsymbol{R}_{u}, \hat{\boldsymbol{\theta}}\right)}{\partial w_{u}}+\frac{\partial^{2} \phi}{\partial w_{u}^{2}} \delta w \\
+\frac{\partial^{2} \phi}{\partial w_{u} \partial \boldsymbol{R}_{u}} \delta \boldsymbol{R}+\frac{\partial^{2} \phi}{\partial w_{u} \partial \boldsymbol{\theta}} \delta \boldsymbol{\theta}
\end{array}
$$

and after subtracting (29)

$$
\frac{\partial^{2} \phi}{\partial w_{u}^{2}} \delta w+\frac{\partial^{2} \phi}{\partial w_{u} \partial \boldsymbol{R}_{u}} \delta \boldsymbol{R}+\frac{\partial^{2} \phi}{\partial w_{u} \partial \boldsymbol{\theta}} \delta \boldsymbol{\theta} \approx 0
$$

and, approximately

$$
\delta w=-\boldsymbol{H}^{-1}\left\{\frac{\partial^{2} \phi}{\partial w_{u} \partial \boldsymbol{R}_{u}} \delta \boldsymbol{R}+\frac{\partial^{2} \phi}{\partial w_{u} \partial \boldsymbol{\theta}} \delta \boldsymbol{\theta}\right\}
$$

where

$$
\boldsymbol{H}=\left[\frac{\partial^{2} \phi}{\partial w_{u}^{2}}\right] .
$$

Equation (33) relates the error in $\hat{w}_{u}(\delta w)$ to the errors in the power ratios $\left(\delta \boldsymbol{R}_{u}\right)$ and the parameter estimates $(\delta \boldsymbol{\theta})$. The covariance matrix of $\hat{w}_{u}$ is equal to the expected or average value of $\left(\delta \boldsymbol{w}_{u}, \delta \boldsymbol{w}_{u}^{\prime}\right)$. The estimated covariance matrix is approximately [4] given by

$$
\begin{aligned}
\boldsymbol{\Sigma}_{w_{u}}= & -\boldsymbol{H}^{-1}\left\{\hat{\sigma}^{2}\left[\frac{\partial^{2} \phi}{\partial w_{u} \partial \boldsymbol{R}_{u}}\right] \boldsymbol{V}_{u}\left[\frac{\partial^{2} \phi}{\partial w_{u} \partial \boldsymbol{R}_{u}}\right]^{\prime}\right. \\
& \left.+\left[\frac{\partial^{2} \phi}{\partial w_{u} \partial \boldsymbol{\theta}}\right] \boldsymbol{\Sigma}_{\theta}\left[\frac{\partial^{2} \phi}{\partial w_{u} \partial \boldsymbol{\theta}}\right]^{\prime}\right\} \boldsymbol{H}^{-1}
\end{aligned}
$$

where $\hat{\sigma}^{2}$ is given in (14) and $\boldsymbol{\Sigma}_{\theta}$ in (16) and

$$
\boldsymbol{V}_{u}=\left[\begin{array}{ccc}
\left(R_{1 u}+.01\right)^{2} & 0 & 0 \\
0 & \left(R_{2 u}+.01\right)^{2} & 0 \\
0 & 0 & \left(R_{3 u}+.01\right)^{2}
\end{array}\right]
$$

\section{C. $w-\Gamma$ Transformation}

The $w-\Gamma$ transform [1] is given by

$$
w=\frac{\alpha \Gamma+\beta}{\gamma \Gamma+1}
$$


where $\alpha, \beta$, and $\gamma$ are the Error-Box parameters. We have estimates of these given in $\hat{\xi}$, and an associated covariance matrix $\boldsymbol{\Sigma}_{\xi}$. The estimated value of $\Gamma$ is obtained by solving (37) for $\Gamma$ which gives

$$
\hat{\Gamma}_{u}=\frac{\hat{w}_{u}-\hat{\beta}}{\hat{\alpha}-\hat{w}_{u} \hat{\gamma}}
$$

where $\hat{w}_{u}$ was obtained in the previous section.

\section{Uncertainty of $\hat{\Gamma}_{u}$}

The uncertainty will be a function of errors in $\hat{w}_{u}$ and $\hat{\xi}$. Thus letting $\hat{\Gamma}_{u}=f\left(\hat{w}_{u}, \hat{\xi}\right)$, where the form of $f$ is in (38), and

$$
\begin{aligned}
& \boldsymbol{J}_{w}=\frac{\partial f}{\partial \hat{w}_{u}} \\
& \boldsymbol{J}_{\xi}=\frac{\partial f}{\partial \hat{\xi}}
\end{aligned}
$$

then, to linear approximations, the covariance matrix of $\hat{\Gamma}_{u}$ is

$$
\boldsymbol{\Sigma}_{\Gamma_{u}}=\boldsymbol{J}_{w}^{\prime} \boldsymbol{\Sigma}_{w_{u}} \boldsymbol{J}_{w}+\boldsymbol{J}_{\xi}^{\prime} \boldsymbol{\Sigma}_{\xi} \boldsymbol{J}_{\xi} .
$$

The expression in (40) does not account for any correlations between $\hat{w}_{u}$ and $\hat{\xi}$. The reason these would be correlated is because they are both obtained from the same value $\hat{\boldsymbol{\theta}}$. However, experimental evidence suggests that these correlations will not significantly affect the estimate in (40), and are therefore not accounted for in the present solution.

At this point (38) represents an estimate of $\Gamma$, and (40) the estimated covariance. Letting $x$ and $y$ denote the real and imaginary parts of a complex number, respectively, we can write

$$
\hat{\Gamma}_{u}=\left[\begin{array}{l}
\Gamma_{x} \\
\Gamma_{y}
\end{array}\right]
$$

and

$$
\boldsymbol{\Sigma}_{\Gamma_{u}}=\left[\begin{array}{ll}
s_{x}^{2} & s_{x y} \\
s_{x y} & s_{y}^{2}
\end{array}\right]
$$

where $s_{x}^{2}$ is the variance of $\Gamma_{x}, s_{y}^{2}$ is the variance $\Gamma_{y}$, and $s_{x y}$ is the covariance between $\Gamma_{x}$ and $\Gamma_{y}$. Conversion to polar coordinates and the application of propagation of error formulae [5] give the magnitude and phase of $\hat{\Gamma}_{u}$ and their associated standard deviations.

\section{Measurement of $S$ Parameters}

When an unknown two-port is inserted butween the two six-ports we obtain the $w$ values for the different excitations from the $P-w$ transformation. From these observed $w$ 's we obtain estimates of the scattering parameters $C$ using the methods outlined in Section III.C. These scattering parameters describe a two-port that consists of the cascade combination of the two Error Boxes and the unknown two-port. The covariance matrix $\boldsymbol{\Sigma}_{C}$ provides an estimate of the errors in the determination of $\boldsymbol{C}$. The scattering parameters for the unknown, $S_{u}$, can be obtained from a functional relationship involving $\boldsymbol{C}$, and the ErrorBox parameters, $\hat{\xi}_{2}$ and $\hat{\xi}_{2}$. We write this as

$$
\boldsymbol{S}_{u}=f\left(\boldsymbol{C}, \hat{\xi}_{1}, \hat{\xi}_{2}\right)
$$

and we obtain the covariance matrix of $S_{u}$ from propagation of error formulae. The attenuation is defined as -10 $\log \left|S_{12}\right|^{2}$ (where $S_{12}$ is an element in $S$ ) and further application of propagation of error formulas give the standard deviation of the attenuation.

\section{Summary}

The measurement accuracy provided by the six-port is a function of the parameter value being measured, the 11 parameters that characterize the six-port, and the errors in their determination. The statistical methods used to estimate the six-port parameters and their uncertainties have been discussed. The measurement of unknown $\Gamma$ or $S$ parameters is obtained by the successive application of the $P-w$ and $w-\Gamma$ transforms, and the uncertainty of these measurements from propagation of error formulas.

\section{Appendix A}

Given the model

$$
Y=f(\theta)+e
$$

where
$Y$ vector of $n$ observations,
$f$ vector of functions,
$\boldsymbol{\theta}$ vector of $p$ parameters, and
$\boldsymbol{e}$ vector of errors with $\operatorname{cov}(\boldsymbol{e})=\sigma^{2} \boldsymbol{V}$.

The least squares estimates are obtained by minimizing a "weighted" sum of squares given by the objective function

$$
\phi(\theta)=e^{\prime} V^{-1} e=(Y-f(\theta))^{\prime} V^{-1}(Y-f(\theta)) .
$$

The minimum is found as the solution to

$$
\frac{\partial \phi(\theta)}{\partial \theta}=\boldsymbol{g}(\boldsymbol{\theta})=0
$$

where $\boldsymbol{g}(\boldsymbol{\theta})$ is the gradient.

The solution to (A3) when the equations are nonlinear is obtained by a Taylor series expansion ignoring 'secondorder terms of $\boldsymbol{g}(\boldsymbol{\theta})$ about $\boldsymbol{\theta}_{i}$ which represents the initial estimate. This gives

$$
g(\theta) \approx \boldsymbol{g}\left(\theta_{i}\right)+\left.\frac{\partial g(\theta)}{\partial \theta}\right|_{\theta_{i}}\left(\theta-\theta_{i}\right)
$$

and equating this to zero and solving for $\theta$ we get

$$
\boldsymbol{\theta}=\boldsymbol{\theta}_{i}-\boldsymbol{H}_{i}^{-1} \boldsymbol{g}\left(\boldsymbol{\theta}_{i}\right)
$$

where

$$
\boldsymbol{H}_{i}=\left.\frac{\partial g(\boldsymbol{\theta})}{\partial \boldsymbol{\theta}}\right|_{\theta_{i}}
$$


We then set $\boldsymbol{\theta}_{i+1}$ to the value obtained in (A5) and evaluate (A5) using $\boldsymbol{\theta}_{i+1}$ as the new value. We iterate until $\left|\theta_{i+1, j}-\theta_{i, j}\right|<\epsilon_{j}$ for all $j$ where

$$
\epsilon_{j}=\frac{\theta_{i, j}+10^{-3}}{10^{4}}
$$

\section{APPEndix B}

The parameter estimates are a function of the observations which are affected by random errors. Of interest to us is the error of estimation that results from errors in the observed data. Such information is contained in the covariance matrix of the parameter estimates.

The objective function that is minimized (A2) depends on the observed data $Y$ and we therefore write $\phi(\theta, Y)$ to reflect this dependence. At the minimum we must have

$$
\frac{\partial \phi(\hat{\boldsymbol{\theta}}, \boldsymbol{Y})}{\partial \boldsymbol{\theta}}=0
$$

However, suppose we varied the data slightly by $\boldsymbol{Y}+\delta \boldsymbol{Y}$; then, this would cause our minimum to shift from $\hat{\boldsymbol{\theta}}$ to $\hat{\boldsymbol{\theta}}$ $+\delta \boldsymbol{\theta}$ where we must also have

$$
\frac{\partial \phi(\hat{\boldsymbol{\theta}}+\delta \hat{\boldsymbol{\theta}}, \boldsymbol{Y}+\delta \boldsymbol{Y})}{\partial \boldsymbol{\theta}}=0 .
$$

Expanding (B2) in Taylor series retaining only first-order terms gives

$\frac{\partial \phi(\hat{\boldsymbol{\theta}}+\delta \hat{\boldsymbol{\theta}}, \boldsymbol{Y}+\delta \boldsymbol{Y})}{\partial \boldsymbol{\theta}} \approx \frac{\partial \phi(\hat{\boldsymbol{\theta}}, \boldsymbol{Y})}{\partial \boldsymbol{\theta}}+\frac{\partial^{2} \phi}{\partial \boldsymbol{\theta}^{2}} \delta \boldsymbol{\theta}+\frac{\partial^{2} \phi}{\partial \boldsymbol{\theta} \partial \boldsymbol{Y}} \delta \boldsymbol{Y}$

and after subtracting (B1)

$$
\frac{\partial^{2} \phi}{\partial \boldsymbol{\theta}^{2}} \delta \boldsymbol{\theta}=-\frac{\partial^{2} \phi}{\partial \boldsymbol{\theta} \partial \boldsymbol{Y}} \delta \boldsymbol{Y}
$$

so that approximately

$$
\delta \boldsymbol{\theta}=-\boldsymbol{H}^{-1}\left\{\frac{\partial^{2} \phi}{\partial \boldsymbol{\theta} \partial \boldsymbol{Y}} \delta \boldsymbol{Y}\right\}
$$

where

$$
\boldsymbol{H}=\left[\frac{\partial^{2} \phi}{\partial \theta^{2}}\right]
$$

Equation (B5) relates the error in $\hat{\boldsymbol{\theta}},(\delta \boldsymbol{\theta})$, to the errors in the observations $(\delta \boldsymbol{Y})$. The covariance matrix of $\hat{\boldsymbol{\theta}}$ is the expected or average value of $\left(\delta \boldsymbol{\theta}, \delta \boldsymbol{\theta}^{\prime}\right)$. The estimated covariance matrix is approximately [4] given by

$$
\Sigma_{\boldsymbol{\theta}}=\hat{\sigma}^{2} \boldsymbol{H}^{-1}\left\{\left[\frac{\partial^{2} \phi}{\partial \boldsymbol{\theta} \partial \boldsymbol{Y}}\right] \boldsymbol{V}\left[\frac{\partial^{2} \phi}{\partial \boldsymbol{\theta} \partial \boldsymbol{Y}}\right]^{\prime}\right\} \boldsymbol{H}^{-1}
$$

where $\hat{\sigma}^{2}$ is

$$
\hat{\sigma}^{2}=\frac{(\boldsymbol{Y}-f(\hat{\boldsymbol{\theta}}))^{\prime} \boldsymbol{V}^{-1}(\boldsymbol{Y}-f(\hat{\boldsymbol{\theta}}))}{n-p} .
$$

In most instances the second partial derivatives can be approximated by

$$
\boldsymbol{H} \approx 2\left(\boldsymbol{J}^{\prime} \boldsymbol{V}^{-1} \boldsymbol{J}\right)
$$

and

$$
\frac{\partial^{2} \phi}{\partial \boldsymbol{\theta} \partial \boldsymbol{Y}} \approx-2\left(\boldsymbol{J}^{\prime} \boldsymbol{V}^{-1}\right)
$$

where

$$
\boldsymbol{J}=\left[\frac{\partial \boldsymbol{f}(\boldsymbol{\theta})}{\partial \boldsymbol{\theta}}\right] .
$$

Using these approximations in (B7) gives

$$
\Sigma_{\theta} \approx \hat{\sigma}^{2}\left(J^{\prime} V^{-1} J\right)^{-1} \text {. }
$$

\section{ACKNOWLEDGMENT}

The authors would like to thank Dr. P. T. Boggs and D. F. Vecchia of the Center for Applied Mathematics, National Bureau of Standards, for their collaboration in the early stages of this effort.

\section{REFERENCES}

[1] G. F. Engen, "On-line accuracy assessment for the dual six-port ANA: Background and theory," pp. 501-506, this issue.

[2] J. Mandel, The Statistical Analysis of Experimental Data. New York: Wiley, 1964, ch. 4, pp. 62-63.

[3] G. F. Engen, "A least squares solution for use in the six-port measurement technique," IEEE Trans. Microwave Theory Tech., vol. MTT-28, pp. 1473-1477, Dec. 1980.

[4] Y. Bard, Nonlinear Parameter Estimation. New York: Academic, 1974, ch. 7, pp. 176-177.

[5] H. H. Ku, "Notes on the use of propagation of error formulas," $J$. Res. Nat. Bur. Stands., vol. 70c, no. 4, Oct.-Dec. 1966; also Nat. Bur. Stand. Special Publ. 300-i, Feb. 1969. 